\title{
Influence of band and orbital degeneracies on ferromagnetism in the periodic Anderson model
}

\author{
Unjong Yu, ${ }^{1,2}$ Krzysztof Byczuk, ${ }^{1,3}$ and Dieter Vollhardt ${ }^{1}$ \\ ${ }^{1}$ Theoretical Physics III, Center for Electronic Correlations and Magnetism, Institute of Physics, University of Augsburg, \\ D-86135 Augsburg, Germany \\ ${ }^{2}$ Department of Physics and Astronomy, Louisiana State University, Baton Rouge, Louisiana 70803, USA \\ ${ }^{3}$ Institute of Theoretical Physics, University of Warsaw, ul. Hoża 69, 00-681 Warszawa, Poland
}

(Received 1 August 2008; published 24 November 2008)

\begin{abstract}
We investigate the influence of degeneracies of the conduction band and the $f$ orbital on the stability of ferromagnetism in the periodic Anderson model. To this end we calculate the temperature dependence of the inverse susceptibility for different degeneracies, $D^{c}$ and $D^{f}$, and conduction-electron densities $n^{c}$ within the dynamical mean-field theory. A strong increase in the Curie temperature $T_{C}$ with the degeneracy $D^{f}$ of the localized $f$ level is found. For $D^{c} \leq D^{f}$ a simple ansatz based on a mean-field treatment of the Ruderman-KittelKasuya-Yoshida interaction is shown to imply a scaling behavior of $T_{C}$ as a function of the conduction-electron density per band which is well obeyed by the numerical results. In particular, $T_{C}$ is found to have a maximum at $n^{c} / D^{c} \approx 0.3$.
\end{abstract}

DOI: 10.1103/PhysRevB.78.205118

PACS number(s): 71.10.Fd, 75.10.-b, 75.20.Hr

\section{INTRODUCTION}

The periodic Anderson model (PAM) is the minimal model for the investigation of interacting localized electrons (e.g., $f$ electrons) hybridizing with a noninteracting band of conduction electrons as in the rare earths and actinides. Indeed, the PAM can explain many of the characteristic properties of heavy fermion systems, ${ }^{1}$ intermediate valence materials, ${ }^{1,2}$ and Kondo insulators. ${ }^{3}$ While it is well known that the PAM can account for the long-range antiferromagnetic correlations at and near half filling of the conduction band, ferromagnetic phases of this model have received less attention. Nevertheless, ferromagnetism induced by localized $^{4-9}$ and even slightly delocalized ${ }^{10} f$ electrons is now known to be a generic property of this model away from half filling. In fact, there exist a number of $f$-electron materials with ferromagnetic phases, e.g., $\mathrm{UCu}_{2} \mathrm{Si}_{2},{ }^{11} \mathrm{UCu}_{2} \mathrm{Ge}_{2},{ }^{11}$ $\mathrm{CeRh}_{3} \mathrm{~B}_{2},{ }^{12} \mathrm{YbNiSn},{ }^{13} \mathrm{URhSi},{ }^{14} \mathrm{URhGe},{ }^{14}$ and CeRuPO.${ }^{15}$ Since the ions providing the $f$ electrons in these systems are rather far apart, their hybridization with conduction electrons must be considered essential for the stabilization of ferromagnetism. This makes the PAM an appropriate model for the investigation of these systems.

The conventional PAM does not take into account any degeneracies of the electrons, e.g., the fact that without crystal-field splitting the $f$ orbitals have a sevenfold degeneracy. This degeneracy can be expected to influence the magnetic properties of materials. For example, in the case of the Hubbard model band degeneracy is known to greatly enhance ferromagnetism due to presence of Hund's rule couplings. ${ }^{16-18}$ Therefore, a more realistic study of ferromagnetism in the PAM should explicitly consider band and orbital degeneracies of the electrons. ${ }^{19}$ In this paper, we present a detailed study of the influence of band degeneracy of the conduction band and/or orbital degeneracy of the localized levels on the stability of ferromagnetism in the PAM for different conduction-electron densities. For this purpose we employ the dynamical mean-field theory (DMFT) with quantum Monte Carlo (QMC) as the impurity solver. In particular, we show that and explain why $T_{C}$ increases with the degeneracy of the $f$ level. The paper is organized as follows. In Sec. II we introduce the model and the calculation method in detail. The result of the magnetization, magnetic susceptibility, and phase diagrams in the PAM with degeneracy are presented and discussed in Sec. III. A summary is presented in Sec. IV.

\section{MODEL AND METHOD OF CALCULATION}

The multiorbital PAM investigated here has the form,

$$
\begin{aligned}
H= & -\sum_{\langle i, j\rangle l \sigma} t_{l}^{c} c_{i l \sigma}^{\dagger} c_{j l \sigma}+\sum_{i l \sigma} \varepsilon_{l}^{c} c_{i l \sigma}^{\dagger} c_{i l \sigma}+\sum_{i m \sigma} \varepsilon_{m}^{f} f_{i m \sigma}^{\dagger} f_{i m \sigma} \\
& +U \sum_{i m} n_{i m\rceil}^{f} n_{i m \downarrow}^{f}+\sum_{i \sigma_{1} \sigma_{2}} \sum_{m_{1}<m_{2}}\left(U^{\prime}-\delta_{\sigma_{1} \sigma_{2}} F\right) n_{i m_{1} \sigma_{1}}^{f} n_{i m_{2} \sigma_{2}}^{f} \\
& +V \sum_{i \sigma} \sum_{l m}\left(c_{i l \sigma}^{\dagger} f_{i m \sigma}+\text { H.c. }\right),
\end{aligned}
$$

where $c_{i l \sigma}^{\dagger}\left(f_{i m \sigma}^{\dagger}\right)$ creates conduction (localized) electrons with orbital $l(m)$ and spin $\sigma$ at site $i$. Furthermore, $t_{l}^{c}$ is the hopping parameter of the conduction electron, while $\varepsilon_{l}^{c}$ and $\varepsilon_{m}^{f}$ denote the center of the conduction band and the energy level of the $f$ electrons, respectively. The orbital index can take the values $l=1, \ldots, D^{c}$ for the conduction band and $m$ $=1, \ldots, D^{f}$ for the $f$ level. In this paper, we consider only degenerate bands, i.e., $t_{l}^{c} \equiv t^{c}, \varepsilon_{l}^{c} \equiv \varepsilon^{c}$, and $\varepsilon_{m}^{f} \equiv \varepsilon^{f}$. Furthermore, $U$ and $U^{\prime}$ are the intraorbital and interorbital Coulomb repulsion, respectively, $F$ is the Ising component of Hund's rule coupling, and $V$ represents the hybridization between the conduction band and the localized orbital. For the conduction band we assume a Bethe density of states (DOS) with $\rho_{c}(\varepsilon)=(1 / 2 \pi) \sqrt{4-\varepsilon^{2}}$ per spin. The bandwidth of the conduction band $(W=4)$ defines the energy scale. We note that the magnetic properties of the PAM do not seem to depend on the specific shape of the noninteracting DOS since a Gaussian DOS gives qualitatively similar results. ${ }^{6}$ In this paper we fix the interactions, the hybridization, and the chemical po- 
tential at the values $U=1.5, U^{\prime}=1.1, F=0.2, V=0.6$, and $\mu$ $=\varepsilon^{f}+U / 2+\left(D^{f}-1\right)\left(U^{\prime}-F / 2\right)$, respectively. For the latter choice of $\mu$, the $f$ level is approximately half filled, ${ }^{22}$ thus permitting local moments to be formed.

In the local moment regime of the PAM ferromagnetism is due to the magnetic interaction between the localized magnetic moments mediated by the noninteracting conduction electrons. While a strong hybridization increases the magnetic interaction, it simultaneously weakens the magnetic moments. For this reason, static mean-field theories or perturbative approaches do not describe ferromagnetic solutions of the PAM adequately. Here the DMFT (Ref. 23) has proved to be a reliable nonperturbative investigation scheme. The DMFT is based on the limit of infinite dimensions of the lattice, where the many-body problem reduces to a local one and can, in principle, be solved exactly. ${ }^{24}$

The structure of the DMFT self-consistency equations for the PAM is the same as that for the Hubbard model, except for the calculation of the lattice Green function through the k-integrated Dyson equation, ${ }^{25}$ which now reads

$$
G_{l \sigma}^{c}\left(\omega_{n}\right)=\frac{1}{N_{k}} \sum_{\mathbf{k}} \frac{1}{i \omega_{n}-\varepsilon_{l}^{c}-\varepsilon_{l \mathbf{k}}-\sum_{m} \frac{V^{2}}{i \omega_{n}-\varepsilon^{f}-\Sigma_{m \sigma}\left(\omega_{n}\right)}},
$$

$$
G_{m \sigma}^{f}\left(\omega_{n}\right)=\frac{1}{N_{k}} \sum_{\mathbf{k}} \frac{1}{i \omega_{n}-\varepsilon^{f}-\Sigma_{m \sigma}\left(\omega_{n}\right)-\sum_{l} \frac{V^{2}}{i \omega_{n}-\varepsilon_{l}^{c}-\varepsilon_{l \mathbf{k}}}} .
$$

Here $\Sigma_{m \sigma}\left(\omega_{n}\right)$ is the self-energy of the $f$ electron with orbital $m$ and spin $\sigma$ and $N_{k}$ is the number of $\mathbf{k}$ points in the summation. The Green functions are represented as a function of the Matsubara frequency $\omega_{n}=(2 n+1) \pi / \beta$, where $\beta=1 / k_{B} T$. Then, the bath Green function is determined by the selfconsistency condition $\mathcal{G}_{m \sigma}^{-1}\left(\omega_{n}\right)=\Sigma_{m \sigma}\left(\omega_{n}\right)+\left[G_{m \sigma}^{f}\left(\omega_{n}\right)\right]^{-1}$, and the Green function is obtained by solving the effective single-impurity problem using QMC. ${ }^{26}$ This QMC method involves a time discretization $\Delta \tau=\beta / L$ and subsequent extrapolation $\Delta \tau \rightarrow 0$. In the present work we do not extrapolate in every case, and mostly work with $\Delta \tau=0.25$ because we are mainly interested in the qualitative behavior of $T_{C}$. As will be shown later the value of $T_{C}$ calculated with $\Delta \tau$ $=0.25$ is within a few percent of the value obtained by the extrapolation $\Delta \tau \rightarrow 0$. For small $U$ (smaller than the bandwidth) the resulting error is small. As $U$ increases, the error increases correspondingly.

The magnetic susceptibility was calculated from the twoparticle correlation function. ${ }^{27}$ It can also be obtained from the magnetization in a weak magnetic field. We found that both methods give the same results although for the same computational time the statistical error in the latter method is much larger.
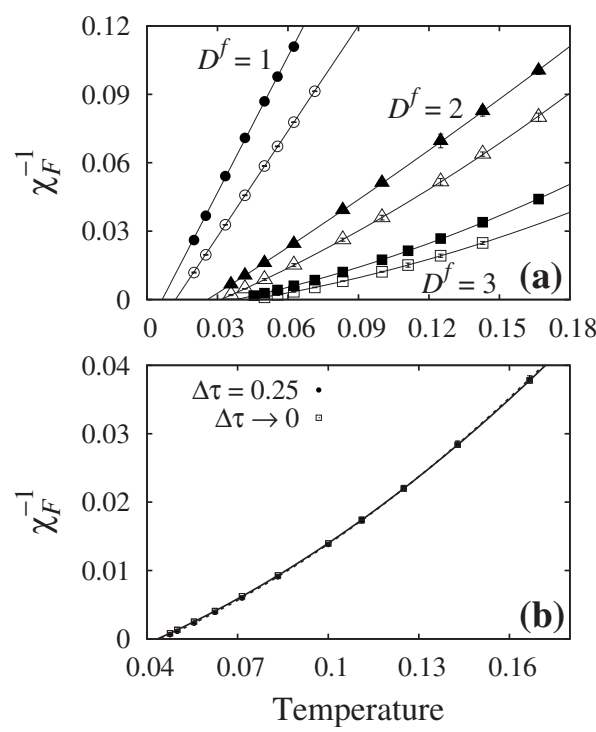

FIG. 1. (a) Inverse susceptibility $\chi_{F}^{-1}$ of the PAM as a function of temperature for three values of the $f$-level degeneracy $D^{f}=1$ (circles), $D^{f}=2$ (triangles), and $D^{f}=3$ (squares) and two values of the conduction-electron density $n^{c}=0.3$ (open symbols) and $n^{c}$ $=0.5$ (filled symbols); the conduction-band degeneracy is $D^{c}=1$ in all cases. Lines were obtained by fitting to Eq. (4). Temperature is in energy units given by the bandwidth $W=4$. (b) Inverse susceptibility $\chi_{F}^{-1}$ calculated with $\Delta \tau=0.25$ compared with the extrapolated result $(\Delta \tau \rightarrow 0)$ for $D^{f}=3$ and $n^{c}=0.4$.

\section{RESULTS AND DISCUSSION}

The numerical results obtained for the inverse ferromagnetic susceptibility $\chi_{F}^{-1}$ of the PAM for values of the $f$-level degeneracy $D^{f}=1,2,3$ and electron densities $n^{c}=0.3,0.5$ are shown in Fig. 1(a). In the nondegenerate case $\left(D^{f}=1\right)$, the inverse susceptibility is found to be almost perfectly linear for all temperatures. A linear behavior of the inverse susceptibility near $T_{C}$, i.e., a Curie-Weiss mean-field behavior, does not come unexpected in DMFT although its validity was so far proved only for the single-band Hubbard model. ${ }^{28}$ The numerical finding that this linear behavior persists up to high temperatures, i.e., that the Curie-Weiss behavior merges into a Curie law, is also not surprising in view of the low values of $T_{C}$ and a saturated magnetization in the magnetic phase.

For increasing degeneracy $D^{f}$, the inverse susceptibility becomes more and more nonlinear. As can be seen from Fig. $1(\mathrm{~b})$, this is not due to the finite value of $\Delta \tau$ since an extrapolation $\Delta \tau \rightarrow 0$ does not change the result. A nonlinear behavior of the susceptibility is also seen in the orbitally degenerate Hubbard model. ${ }^{29}$ This nonlinear behavior, i.e., the change in slope with temperature, is due to the decrease in the effective magnetic moment $\mu_{\text {eff }}=\sqrt{\chi_{F}\left(T-T_{C}\right)}$ with temperature. This effect is negligible in the nondegenerate case $\left(D^{f}=1\right)$. By contrast, in the degenerate case the spins of the localized electrons are aligned parallel due to Hund's rule coupling near $T_{C}$ (which is here found to be low) such that the effective magnetic moment becomes large. On the other hand, at high temperatures the effective magnetic moment decreases since the electrons become independent and the spin of the localized electron takes a random orientation on 


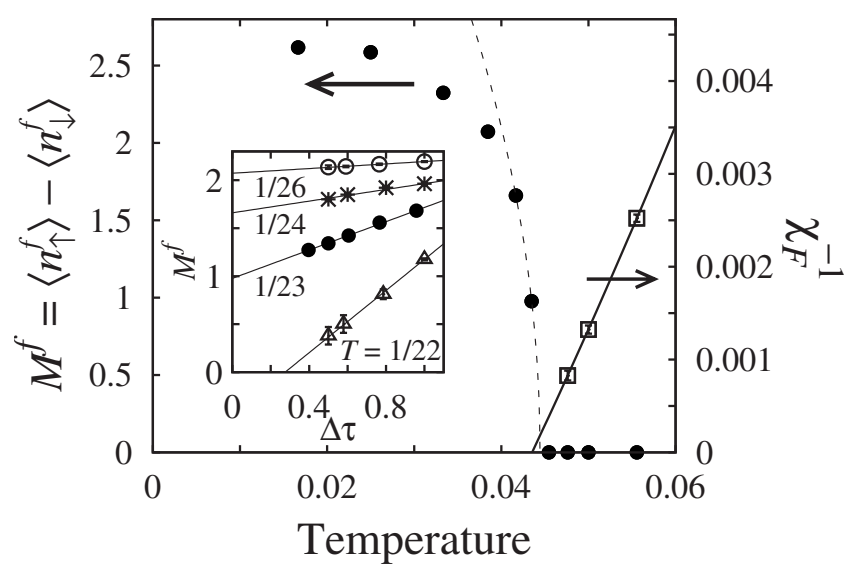

FIG. 2. Magnetization and inverse susceptibility $\chi_{F}^{-1}$ extrapolated to $\Delta \tau \rightarrow 0$ as a function of temperature for $D^{c}=1, D^{f}=3$, and $n^{c}=0.4$. The dashed line marks a rootlike disappearance of the magnetization fitted to the last two data points in the magnetic phase. The inset shows the $\Delta \tau$ dependence of the magnetization for temperatures $T=1 / 26,1 / 24,1 / 23$, and $1 / 22$.

every site, owing to the thermal fluctuation. Accordingly, the decrease in the effective magnetic moment with increasing temperature leads to an increase in the slope of the inverse susceptibility. This explains the upturn of the inverse susceptibility for increasing temperature.

The nonlinearity of $\chi_{F}^{-1}$ has to be taken into account in the calculation of $T_{C}$ from the inverse susceptibility. Namely, a purely linear fitting of $\chi_{F}^{-1}$ would lead to a serious error in the value of $T_{C}$. We found the inverse susceptibility to be well fitted by

$$
\chi_{F}^{-1}=A\left(T-T_{C}\right)+B\left(T-T_{C}\right)^{2} .
$$

The linear behavior is restored again at high temperatures, i.e., above the Fermi temperature (Curie law). The two linear regimes at high temperatures and near $T_{C}$ are smoothly connected.

To check the validity of Eq. (4) and the $T_{C}$ values obtained from that expression, we calculated the magnetization $M^{f}=\left\langle n_{\uparrow}^{f}\right\rangle-\left\langle n_{\downarrow}^{f}\right\rangle$ of the ferromagnetic phase of the degenerate PAM. To reduce the error from thermal fluctuations in the QMC calculation we performed an extrapolation $1 / N_{\mathrm{MC}}$ $\rightarrow 0$, where $N_{\mathrm{MC}}$ is the number of Monte Carlo steps in the $\mathrm{QMC}$ routine, to calculate the magnetization at each iteration. Then we performed an extrapolation $\Delta \tau \rightarrow 0$. As shown in the inset of Fig. 2, the magnetization depends linearly on $\Delta \tau$ and a spontaneous magnetization begins to appear at temperature between $T=1 / 23$ and $1 / 22$. The magnetization and inverse magnetic susceptibility obtained after the extrapolation $\Delta \tau \rightarrow 0$ are presented in Fig. 2. The value of $T_{C}$ obtained by fitting the susceptibility to Eq. (4) is clearly consistent with the values inferred from the magnetization (inset of Fig. 2 ) and from the root fit to the magnetization (dashed curve in Fig. 2).

The dependence of the Curie temperature $T_{C}$ on the conduction-electron density $n^{c}$ is shown in Fig. 3 for several values of the $f$ level $\left(D^{f}\right)$ and conduction-band $\left(D^{c}\right)$ degeneracy. The most remarkable feature is the pronounced in-
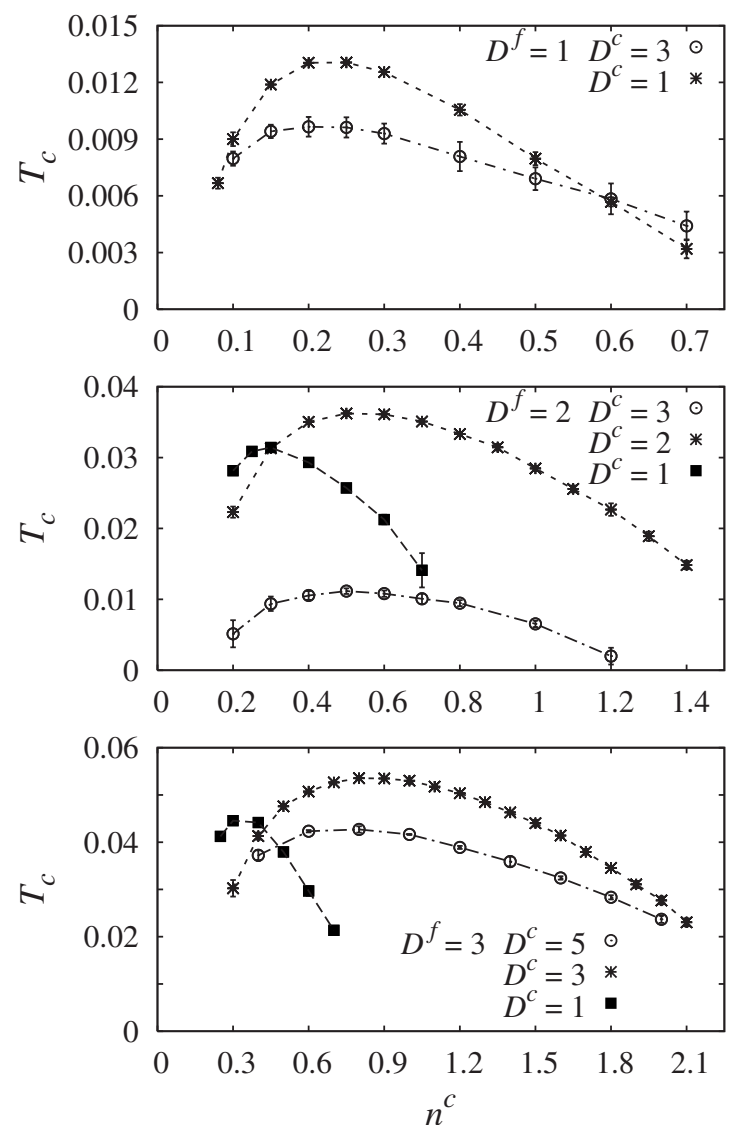

FIG. 3. Curie temperature as a function of conduction-electron density $n_{c}$ for different values of the $f$-level degeneracy $D^{f}$ and conduction-band degeneracy $D^{c}$.

crease in $T_{C}$ with $D^{f}$. This behavior can be explained by the increase in the local magnetic moment. Namely, Hund's rule coupling aligns the local magnetic moments on each site, whereby the magnitude of the local moment, $S$, increases with the degeneracy $D^{f} ; T_{C}$ then increases accordingly. By contrast, the degeneracy of the conduction band has a different effect. For increasing $D^{c}$ the conduction-electron density giving the maximal $T_{C}$ (i.e., the "optimal" density) also increases, whereby the ferromagnetic phase expands to higher $n^{c}$ values. At the same time $T_{C}$ is hardly affected. For $D^{c}$ $\geq D^{f}$, however, $T_{C}$ almost always decreases with increasing $D^{c}$.

In order to explain the general dependence of $T_{C}$ on the degeneracy shown in Fig. 3 we modify the ansatz that we recently introduced in the case of the disordered PAM without degeneracy, ${ }^{30}$ which has the form $T_{C}(\mu)=T_{C}^{0} F^{f}(\mu$ $\left.-\varepsilon^{f}\right) F^{c}\left(\mu-\varepsilon^{c}\right)$. Here the two functions, $F^{f}$ and $F^{c}$, describe the formation of the local $f$-electron moments and the mediation of the magnetic ordering by $c$ electrons, respectively. Since $\mu-\varepsilon^{f}$ is fixed, $F^{f}\left(\mu-\varepsilon^{f}\right)$ depends only on the degeneracy. According to the mean-field theory for the RudermanKittel-Kasuya-Yoshida (RKKY) interaction in which this ansatz is indeed justified the local moment part $\left(F^{f}\right)$ is proportional to $J_{\text {eff }}^{2} S(S+1)$, where $J_{\text {eff }}=-8 V^{2} / U_{\text {eff }}{ }^{31}$ The effective Coulomb interaction $U_{\text {eff }}$ is defined as $U_{\text {eff }}=E\left(n_{0}^{f}\right.$ $+1)+E\left(n_{0}^{f}-1\right)-2 E\left(n_{0}^{f}\right)$, where $E\left(n_{0}^{f}\right)$ is the energy with the $f$-electron density $n_{0}^{f} \cdot{ }^{32,33}$ For a half-filled $f$ level, $n_{0}^{f}=D^{f}$ and 


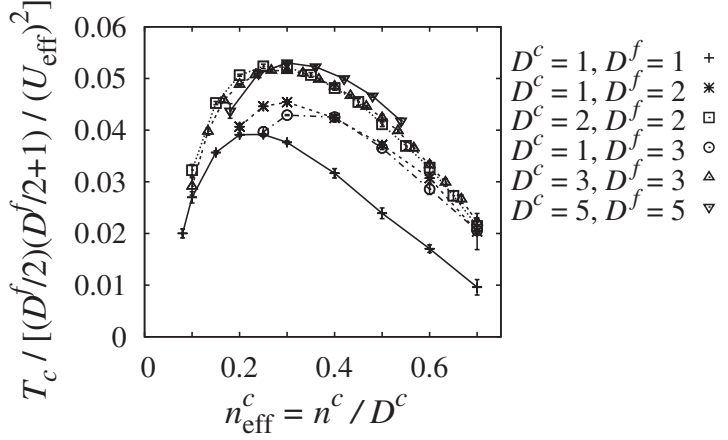

FIG. 4. Rescaled plot of the Curie temperature $T_{C}$ vs conduction-electron density $n_{c}$ for several values of the $f$-level degeneracy $D^{f}$ and conduction-band degeneracy $D^{c}$, with $D^{c}<D^{f}$.

$U_{\text {eff }}=U+\left(D^{f}-1\right) F$. Thus, assuming $S=D^{f} / 2, F^{f}$ is proportional to $\left(D^{f} / 2\right)\left(D^{f} / 2+1\right) /\left[U+\left(D^{f}-1\right) F\right]^{2}$. As in the conduction-band part, $F^{c}\left(\mu-\varepsilon^{c}\right)$ can be expressed by $F^{c}\left(n_{\mathrm{eff}}^{c}\right)$ with $n_{\mathrm{eff}}^{c}=n^{c} / D^{c}$ because $\mu-\varepsilon^{c}$ is uniquely determined by the conduction-electron density per band $\left(n_{\mathrm{eff}}^{c}\right)$. Therefore, when we plot $T_{C} /\left\{\left(D^{f} / 2\right)\left(D^{f} / 2+1\right) /\left[U+\left(D^{f}-1\right) F\right]^{2}\right\}$ as a function of $n^{c} / D^{c}$, all curves should fall onto the same curve. The result is shown in Fig. 4. The deduced scaling behavior is seen to be obeyed surprisingly well, given the simplicity of the assumptions made in the derivation and the fact that the parameter values employed in our investigation are not limited to the RKKY regime. Indeed $T_{C}$ increases rapidly with $n_{\text {eff }}^{c}$ until it reaches a maximum near $n_{\text {eff }}^{c}=0.3$ and then decreases slowly. The maximal value of $T_{C}$ increases with $D^{f}$ and is about $0.05\left\{\left(D^{f} / 2\right)\left(D^{f} / 2+1\right) /\left[U+\left(D^{f}-1\right) F\right]^{2}\right\}$. In accordance with the mean-field character of the above ansatz the curves for $D^{c}>1$ are found to fulfill the scaling behavior much better than for $D^{c}=1$. For $D^{c}>D^{f}$, which are not plotted in Fig. 4, the above ansatz does not apply. The additional conduction bands seem to impede the magnetic ordering rather than mediate it.

\section{CONCLUSION}

We computed the ferromagnetic susceptibility of the PAM and from that the Curie temperature $T_{C}$ for different values of the conduction band and $f$-level degeneracies as well as electron densities $n^{c}$ within DMFT. The magnetic susceptibility was found to deviate from the Curie-Weiss behavior away from $T_{C}$, an effect which becomes stronger with increasing degeneracy of the $f$ level. Our results show that the degeneracy affects the ferromagnetic stability of the PAM strongly. In particular, for $D^{c} \leq D^{f}$ the value of $T_{C}$ (i) increases with the $f$-level degeneracy, (ii) approximately obeys a scaling law as a function of the conduction-electron density per band $n^{c} / D^{c}$, which can be understood within a meanfield picture of the RKKY interaction, and (iii) becomes maximal for $n^{c} / D^{c} \approx 0.3$. This shows that realistic investigations of correlated electron materials with localized $f$ levels must employ appropriate generalizations of the PAM where the multiorbital character of the electrons is taken into account.

\section{ACKNOWLEDGMENTS}

This work was supported in part by the Sonderforschungsbereich 484 of the Deutsche Forschungsgemeinschaft (DFG).
${ }^{1}$ G. Czycholl, Phys. Rep. 143, 277 (1986); P. Schlottmann, ibid. 181, 1 (1989).

${ }^{2}$ C. M. Varma, Rev. Mod. Phys. 48, 219 (1976).

${ }^{3}$ P. S. Riseborough, Adv. Phys. 49, 257 (2000).

${ }^{4}$ A. M. Reynolds, D. M. Edwards, and A. C. Hewson, J. Phys.: Condens. Matter 4, 7589 (1992); B. Möller and P. Wölfle, Phys. Rev. B 48, 10320 (1993); R. Doradziński and J. Spałek, ibid. 58, 3293 (1998).

${ }^{5}$ M. Guerrero and R. M. Noack, Phys. Rev. B 53, 3707 (1996).

${ }^{6}$ A. N. Tahvildar-Zadeh, M. Jarrell, and J. K. Freericks, Phys. Rev. B 55, R3332 (1997).

${ }^{7}$ P. Santini, R. Lémanski, and P. Erdös, Adv. Phys. 48, 537 (1999).

${ }^{8}$ J. W. Rasul, Phys. Rev. B 61, 15246 (2000).

${ }^{9}$ D. Meyer and W. Nolting, Phys. Rev. B 62, 5657 (2000).

${ }^{10}$ C. D. Batista, J. Bonča, and J. E. Gubernatis, Phys. Rev. Lett. 88, 187203 (2002).

${ }^{11}$ L. Chełmicki, J. Leciejewicz, and A. Zygmunt, J. Phys. Chem. Solids 46, 529 (1985).

${ }^{12}$ S. K. Malik, A. M. Umarji, G. K. Shenoy, P. A. Montano, and M. E. Reeves, Phys. Rev. B 31, 4728 (1985).

${ }^{13}$ P. Bonville, P. Bellot, J. A. Hodges, P. Imbert, G. Jéhanno, G. Le Bras, J. Hammann, L. Leylekian, G. Chevrier, P. Thuéry, L.
D’Onofrio, A. Hamzic, and A. Barthélémy, Physica B 182, 105 (1992).

${ }^{14}$ V. H. Tran, R. Troć, and G. André, J. Magn. Magn. Mater. 186, 81 (1998).

${ }^{15}$ C. Krellner, N. S. Kini, E. M. Brüning, K. Koch, H. Rosner, M. Nicklas, M. Baenitz, and C. Geibel, Phys. Rev. B 76, 104418 (2007).

${ }^{16}$ For a review, see D. Vollhardt, N. Blümer, K. Held, M. Kollar, J. Schlipf, M. Ulmke, and J. Wahle, Adv. Solid State Phys. 38, 383 (1999).

${ }^{17}$ A. M. Oleś, Phys. Rev. B 23, 271 (1981); G. Stollhoff and P. Thalmeier, Z. Phys. B: Condens. Matter 43, 13 (1981); G. Stollhoff, A. M. Oleś, and V. Heine, Phys. Rev. B 41, 7028 (1990); R. Frésard and G. Kotliar, ibid. 56, 12909 (1997).

${ }^{18}$ J. Kuei and R. T. Scalettar, Phys. Rev. B 55, 14968 (1997); J. E. Hirsch, ibid. 56, 11022 (1997); M. Fleck, A. M. Oleś, and L. Hedin, ibid. 56, 3159 (1997).

${ }^{19}$ The $\mathrm{SU}(N)$ symmetric PAM, where $N$ is the degeneracy of the $f$ level and the conduction band, can be solved exactly (Ref. 20) for $N \rightarrow \infty$ but does not show a magnetic instability in this limit since the magnetic interaction is suppressed (Ref. 21) as $\sim 1 / N^{2}$.

${ }^{20}$ P. Coleman, Phys. Rev. B 29, 3035 (1984).

${ }^{21}$ D. M. Newns and N. Read, Adv. Phys. 36, 799 (1987). 
${ }^{22}$ Without hybridization this choice of the chemical potential results in a half-filled $f$ level. For nonzero hybridization, this does so only approximately.

${ }^{23}$ For reviews, see A. Georges, G. Kotliar, W. Krauth, and M. Rozenberg, Rev. Mod. Phys. 68, 13 (1996); G. Kotliar and D. Vollhardt, Phys. Today 57(3), 53 (2004).

${ }^{24}$ W. Metzner and D. Vollhardt, Phys. Rev. Lett. 62, 324 (1989).

${ }^{25}$ M. Jarrell, H. Akhlaghpour, and Th. Pruschke, Phys. Rev. Lett. 70, 1670 (1993).

${ }^{26}$ J. E. Hirsch and R. M. Fye, Phys. Rev. Lett. 56, 2521 (1986).

${ }^{27}$ M. Ulmke, V. Janiš, and D. Vollhardt, Phys. Rev. B 51, 10411 (1995).
${ }^{28}$ K. Byczuk and D. Vollhardt, Phys. Rev. B 65, 134433 (2002).

${ }^{29}$ S. Sakai, R. Arita, K. Held, and H. Aoki, Phys. Rev. B 74, 155102 (2006).

${ }^{30}$ U. Yu, K. Byczuk, and D. Vollhardt, Phys. Rev. Lett. 100, 246401 (2008).

${ }^{31}$ This formula can be derived by a multiorbital generalization of the Schrieffer-Wolff transformation; see B. Mühlschlegel, Z. Phys. 208, 94 (1968).

${ }^{32}$ V. I. Anisimov and O. Gunnarsson, Phys. Rev. B 43, 7570 (1991).

${ }^{33}$ V. I. Anisimov, J. Zaanen, and O. K. Andersen, Phys. Rev. B 44, 943 (1991). 